\title{
Percutaneous Thrombectomy for the Treatment of Acute Massive Pulmonary Embolism: Two Case Reports
}

\author{
Kinley Sangay Dorji, M.D. ${ }^{1,2}$, Sorracha Rookkapan, M.D. ${ }^{2}$, Wiwatana Tanomkiat, M.D. ${ }^{2}$, \\ Keerati Hongsakul, M.D. ${ }^{2}$ \\ 'Eastern Regional Referral Hospital, Mongar 43001, Bhutan. \\ ${ }^{2}$ Department of Radiology, Faculty of Medicine, Prince of Songkla University, Hat Yai, Songkhla 90110, Thailand. \\ Received 8 May 2020 • Revised 26 July 2020 • Accepted 5 August 2020 • Published online 15 December 2020
}

\begin{abstract}
:
Pulmonary embolism (PE) is the third commonest cause of death in hospitalized patients after myocardial infarction and stroke. Surgical thrombectomy is a standard option in cases of a clinical massive PE with hemodynamic instability and in patients in whom systemic thrombolysis is contra-indicated. Percutaneous thrombectomy is a new minimally invasive alternative treatment for clinical massive PE, and it has a high efficacy, with fewer complications. We report two patients with acute massive PE that were treated successfully using the endovascular technique with suction thrombectomy.
\end{abstract}

Keywords: endovascular treatment, massive pulmonary embolism, thrombectomy

Contact: Assoc. Prof. Keerati Hongsakul, M.D.

Division of Interventional Radiology, Department of Radiology, Faculty of Medicine, Prince of Songkla University, Hat Yai, Songkhla 90110, Thailand.

E-mail: keerati.hongsakul@yahoo.com

2020 JHSMR. Hosting by Prince of Songkla University. All rights reserved.

This is an open access article under the CC BY-NC-ND license

(http://www.jhsmr.org/index.php/jhsmr/about/editorialPolicies\#openAccessPolicy). 


\section{Introduction}

Acute pulmonary embolism (PE) refers to the sudden obstruction of the pulmonary artery and/or its branches by a thrombus. The symptoms of the acute $\mathrm{PE}$ range from an asymptomatic PE diagnosed incidentally to a massive PE characterized by systemic hypotension or shock. ${ }^{1}$ The acute massive PE is associated with a significant morbidity and mortality, with a 90-day mortality rate exceeding $50.0 \%$. $^{2}$ Surgical thrombectomy is the standard treatment in cases of a massive PE with hemodynamic instability. However, this major operation has high morbidity and mortality rates, particularly in elderly patients. Recently, the endovascular technique using percutaneous thrombectomy (PT) has been used as an alternative option for the treatment of an acute massive PE. The reported technical success rate ranges between $92.0 \%$ and $94.0 \%{ }^{3,4}$ We report two patients suffering from an acute massive PE with hemodynamic instability that were treated successfully via percutaneous suction thrombectomy.

\section{Case reports}

\section{Case 1}

A 60-year-old male, a known case of multiple myeloma awaiting autologous stem cell transplantation, presented to the emergency room with sudden onset dyspnea and lethargy for 1 day. On examination, the patient had mild tachypnea (respiration rate 24/minute) and hypoxemia (SpO2 88.0-90.0\% at room air). He was normotensive, and the other examination findings were unremarkable. The electrocardiography (ECG), troponin-T, and echocardiography results were negative for right ventricular strain. However, the Computed Tomography Angiography (CTA) of the pulmonary artery revealed extensive acute PE in both the right and left main pulmonary arteries (Figure 1A). Moreover, the patient had a low platelet count $(29,000 /$ milliliter $)$. According to the medical consultation conclusions, anticoagulation and systemic thrombolysis were not advised due to thrombocytopenia, and the surgical team recommended that the risks of surgical thrombectomy outweighed its benefits. Hence, the intervention radiologist was consulted for endovascular treatment. The endovascular procedure was performed under general anesthesia. The pre-procedural angiography showed filling defects in both main pulmonary arteries-in both lower lobar and right upper lobar branches (Figure 1B). PT was performed using the Penumbra system [Penumbra, Inc., Alameda, California (CA), The United States of America (USA)], which is a suction thrombectomy system. An 8-French Penumbra INDIGO CAT catheter was used; it was connected to a continuous suction vacuum system to aspirate the thrombi from the right and left main pulmonary arteries. A wire separator was also used to break the thrombi. The post-procedural angiography showed a smaller size of residual thrombi in the right main pulmonary artery and the left lower lobar branch with much improved vascular flow to the segmental branches of both lungs (Figure 1C). Furthermore, no immediate procedure-related compilation was detected. An inferior vena cava filter was also placed due to right femoral deep vein thrombosis (DVT). One day after the procedure, the patient's clinical condition improved, and room air oxygenation was at $98.0 \%$. His follow-up CTA of the pulmonary artery 1 week after the procedure revealed a much-improved extensive acute PE (Figure 1D). Finally, he was planned to be put on enoxaparin therapy when his platelet count improved.

\section{Case 2}

A 75-year-old female, with a history of primary peritoneal cancer, was admitted after colonoscopy detected a complicated sigmoid perforation. She underwent exploratory laparotomy and repair. One week later, the patient developed sudden-onset dyspnea with hypoxemia and hypotension. Her oxygen saturation was $86.0 \%$ at room air, and her blood pressure was $68 / 49 \mathrm{mmHg}$. Her ECG 

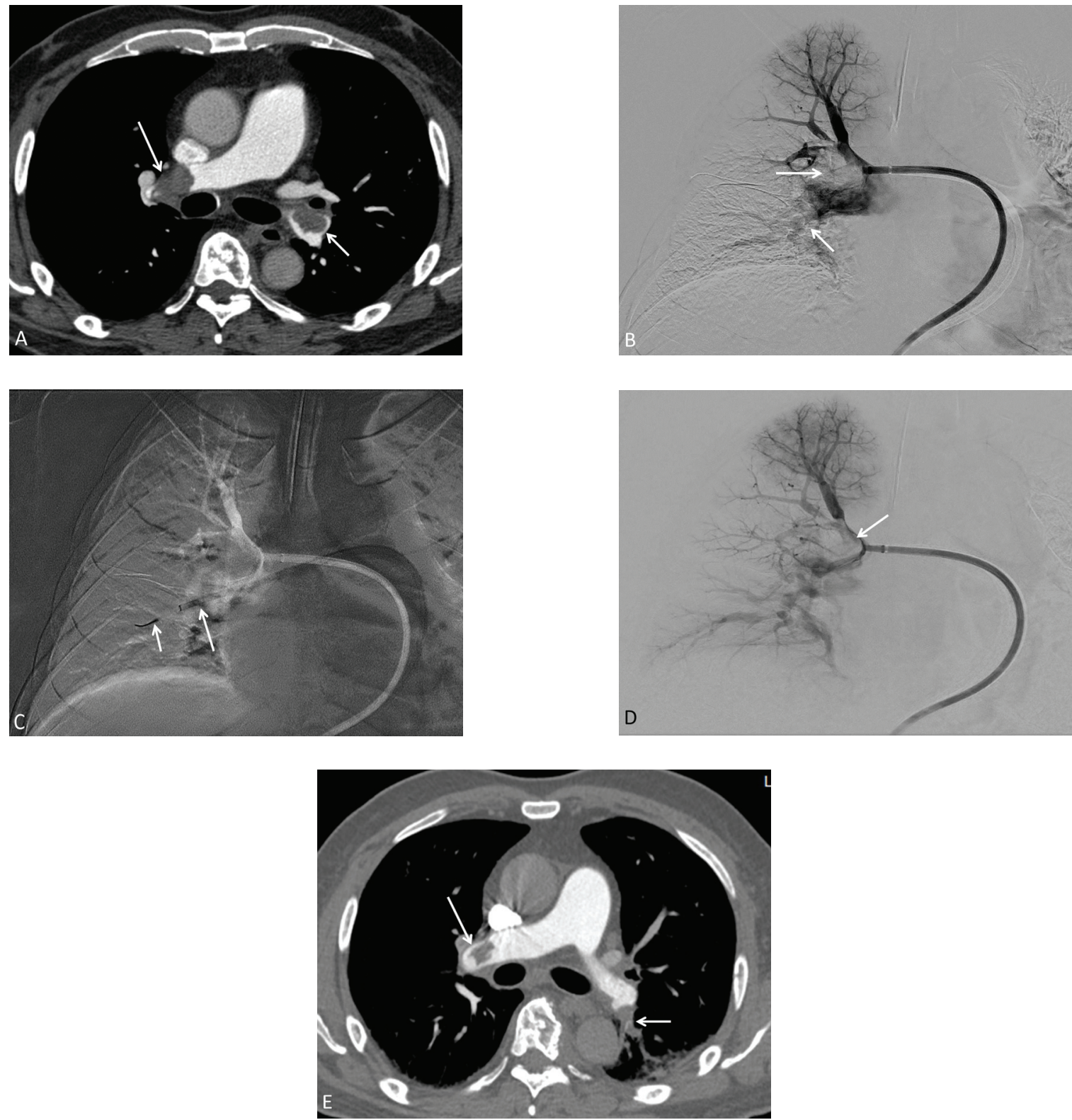

Figure 1 (A) Computed Tomography Angiography of the pulmonary arteries showed acute thrombi in the right (large arrow) and left main pulmonary arteries (small arrow); (B) The right pulmonary angiography revealed acute thrombi in right main pulmonary artery (large arrow) and its lower lobe segmental branch (small arrow); (C) A suction catheter (large arrow) and a wire separator were used during the procedure; (D) The final angiography showed an improved perfusion to the right lung with a residual thrombus in the right main pulmonary artery (arrow); (E) Computed Tomography Angiography of the pulmonary arteries at 1 week after the procedure showed small residual thrombi in the right (large arrow) and left main pulmonary arteries (small arrow). 
showed atrial fibrillation with a right ventricular rate of 129/ minute. The CTA of the pulmonary arteries demonstrated acute massive $\mathrm{PE}$ in the right main pulmonary artery as well as the whole right lobar and left middle and lower lobar branches with right ventricular strain (Figure 2A and 2B). She was immediately anticoagulated, and the cardiovascular team was consulted for surgical thrombectomy. However, the patient refused open surgery; therefore, endovascular treatment became the only option for this patient. The pre-procedural angiogram revealed large filling defects in the right main pulmonary artery as well as the upper, middle and lower lobe branches that were associated with a decreased perfusion of the right lung (Figure 2C) with a minimal pulmonary perfusion defect as well as a rather small thrombus in the segmental branch of the left lower lobe. She was treated with percutaneous suction thrombectomy using the Penumbra system (Penumbra, Inc., Alameda, CA, USA) with an 8-French Penumbra INDIGO CAT catheter. The post-procedural angiogram showed much decreased filling defects in the right main pulmonary artery as well as the middle and lower lobe branches with small amounts of residual thrombi (Figure 2D-F). The procedure's result was excellent; the patient's condition improved, and there were no complications. She was also diagnosed with right popliteal DVT and was treated with heparin infusion.

\section{Discussion}

$\mathrm{PE}$ is the third commonest cause of death in hospitalized patients. ${ }^{5}$ The current standard of practice involves the treatment of massive PE with systemic thrombolysis. ${ }^{6,7}$ However, a common and the main limiting factor for systemic thrombolysis is bleeding, especially intracranial hemorrhage. Most hospitalized patients are severely ill and usually have one or more risk factors for thrombolysis such as coagulopathy or having undergone a recent surgical procedure. In addition, systemic thrombolysis takes longer to act and might not even work in cases of a huge clot burden. Surgical thrombectomy is the standard treatment in cases of massive PE with hemodynamic instability; however, it is associated with a significant mortality rate ranging from $3.6 \%$ to $27.2 \%{ }^{8}$ As a result, many surgeons are reluctant to perform this procedure or keep it as a last option for severe cases, which are usually associated with a poor prognosis. ${ }^{9}$

In our cases, both patients carried a huge clot burden that might have either not responded at all or had a slow response to systemic thrombolysis. In addition, our first patient also had thrombocytopenia, which increases the risk of bleeding after thrombolysis. Moreover, the cardiothoracic surgeons involved in these patients' treatment pointed out that the risks of surgical thrombectomy outweighed its benefits in the first patient, while the second patient preferred minimally invasive endovascular treatment over surgery. Therefore, both patients were treated via PT.

$\mathrm{PT}$ is a minimally invasive procedure for the treatment of massive PE, which involves removing the thrombus from the pulmonary artery and its branches via a catheterbased approach. It provides rapid symptomatic relief with a lower risk of bleeding compared to open surgery. It is also associated with a shorter hospitalization and faster recovery. In addition, this is a viable option for PE cases with a huge clot burden that are otherwise refractory to thrombolysis. However, the major complications of PT include renal failure, bradycardia, stroke, and death; they constitute less than $5.0 \%$ of overall cases. ${ }^{10,11}$

There are two types of mechanism of thrombus removal, which can be broadly divided into mechanical thrombectomy and suction thrombectomy. ${ }^{5}$ Mechanical thrombectomy involves breaking the clot before removing it, which can be done via a simple procedure such as rotating 

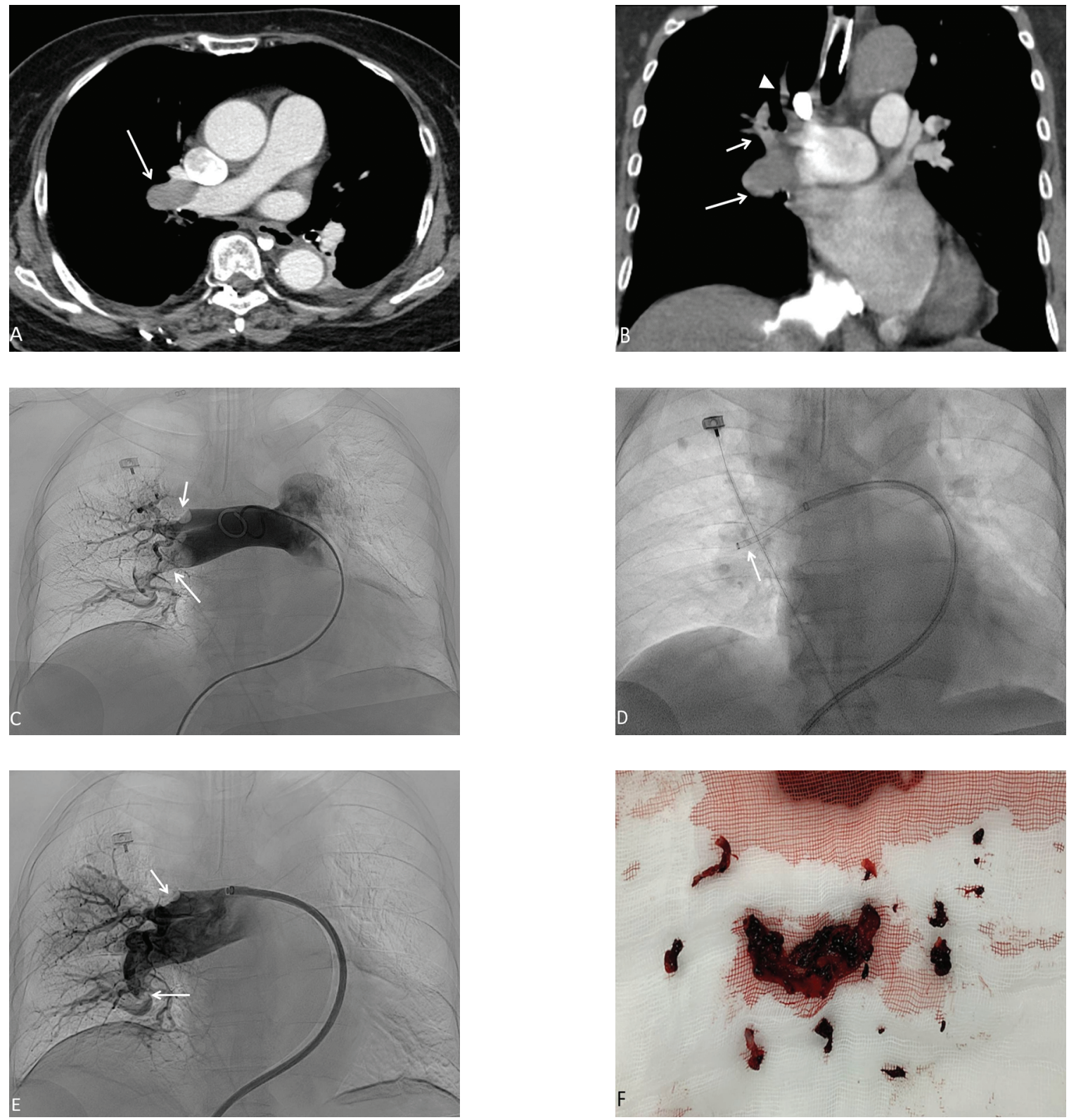

Figure 2 The axial (A) and coronal (B) views of computed tomography angiography of the pulmonary arteries showed acute thrombi in right main pulmonary artery (large arrow) and its upper lobe segmental branch (small arrow and arrowhead); (C) The pulmonary angiography revealed acute thrombi in right main pulmonary artery (large arrow) and its upper lobe segmental branch (small arrow); (D) The suction catheter (arrow) was used to aspirate the thrombus; (E) The final angiography showed an improved perfusion to the right lung with a residual thrombus (arrows); (F) Fragments of thrombus from the right pulmonary artery. 
the catheter within the thrombus, using an angioplasty balloon, or using more sophisticated devices as is the case in rheolytic thrombectomy. The angiojet thrombectomy device (Boston Scientific, Marlborough, Massachusetts, USA) is one of the commonly used rheolytic devices, through which saline solution is directed at high speed towards the clot from the distal end of the catheter in order to break the clot. The fragmented clots are then aspirated. ${ }^{4}$ On the other hand, suction thrombectomy involves the removal of the clot by aspiration without having to break it first. The Angiovac catheter (Angiodynamic, Inc., Latham, New York, USA), the Flowtriever device (Inari Medical, Irvine, CA, USA), and the Pneumbra system (Penumbra, Inc., Alameda, CA, USA) are some of the examples of such devices. ${ }^{4}$ In both of our patients, we used the Penumbra system because it is the only one of the three available in our institution. In addition, the system is easy to use and less traumatic to vessel walls because the tip of the suction catheter is softer than those of the other devices. However, the disadvantage of this device is that it does not allow for the re-circulation of the aspirated blood; therefore, this may cause a large amount of blood volume loss.

Currently, the usefulness of PT in the treatment of massive PE still lacks evidence support from randomizedcontrolled trails. However, some studies, especially case reports, have elaborated on such situations and demonstrated the safety and efficacy of PT in the successful management of massive PE. 10,11 The reported technical success rate has ranged from $92.0 \%$ to $94.0 \% .^{3,4}$ In addition, some studies have shown that PT combined with local fibrinolysis achieves a rapid return to normal pulmonary pressure in a safe and effective way. ${ }^{12}$ With more evidence support from further and larger-scale research studies, PT may become a trustworthy alternative treatment for acute massive PE in the future.

\section{Conclusion}

PT offers a minimally invasive alternative option for the treatment of acute massive PE with good results. It provides rapid symptomatic relief and is associated with a shorter hospital admission.

\section{Conflict of interest}

The authors declare no conflict of interest.

\section{References}

1. Sekhri V, Mehta N, Rawat N, Lehrman SG, Aronow WS. Management of massive and nonmassive pulmonary embolism. Arch Med Sci 2012;8:957-69.

2. Kucher N, Rossi E, De Rosa M, Goldhaber SZ. Massive pulmonary embolism. Circulation 2006;113:577-82.

3. Chechi T, Vecchio S, Spaziani G, Giuliani G, Giannotti F, Arcangeli C, et al. Rheolytic thrombectomy in patients with massive and submassive acute pulmonary embolism. Catheter Cardiovasc Interv 2009;73:506-13.

4. Ciampi-Dopazo JJ. Aspiration thrombectomy for treatment of acute massive and submassive pulmonary embolism: initial single-center prospective experience. J Vasc Interv Radiol 2018;29:101-6

5. Nosher JL, Patel A, Jagpal S, Gribbin C, Gendel V. Endovascular treatment of pulmonary embolism: selective review of available techniques. World J Radiol 2017;9:426-37.

6. Konstantinides SV, Torbicki A, Agnelli G, Danchin N, Fitzmaurice D, Galiè N, et al. 2014 ESC Guidelines on the diagnosis and management of acute pulmonary embolism. Eur Heart J 2014; 35:3033-69.

7. Jaff MR, McMurtry MS, Archer SL, Cushman M, Goldenberg N, Goldhaber SZ, et al. Management of massive and submassive pulmonary embolism, iliofemoral deep vein thrombosis, and chronic thromboembolic pulmonary hypertension: a scientific statement from the American Heart Association. Circulation 2011;123:1788-830.

8. Fukuda I, Daitoku K. Surgical embolectomy for acute pulmonary thromboembolism. Ann Vasc Dis 2017;10:107-14. 
9. Yavuz S, Toktas F, Goncu T, Eris C, Gucu A, Ay D, et al. Surgical embolectomy for acute massive pulmonary embolism. Int $\mathrm{J}$ Clin Exp Med 2014;7:5362-75.

10. Myat A, Ahsan A. Percutaneous mechanical thrombectomy for the treatment of acute massive pulmonary embolism: case report. Thromb J 2007;5:20.
11. Tukaye DN, McDaniel M, Liberman H, Burkin $\mathrm{Y}$, Jaber $\mathrm{W}$. Percutaneous pulmonary embolus mechanical thrombectomy. J Am Coll Cardiol Int 2017;10:94-5.

12. De Gregorio MA, Guirola JA, Lahuerta C, Serrano C, Figueredo AL, Kuo WT. Interventional radiology treatment for pulmonary embolism. World J Radiol 2017;9:295-303. 